\title{
Local and systemic permanent second molar eruption pathology: diagnostic and therapeutic decision trees
}

\author{
H. Guiral-Desnoës \\ DFO specialist, community medical center
}

\begin{abstract}
Permanent second molar eruption anomalies, although rare, seem to have become increasingly frequent over the past decades. The present article first inventories and, when necessary, defines the many local and systemic etiologies. Then two decision trees are described, to help clinicians in case of non-eruption of second molars at the normal age; the first seeks to specify diagnosis, and the second to guide treatment planning.
\end{abstract}

KEYWORDS

Second molar, mechanical failure of eruption, primary failure of eruption

\section{INTRODUCTION}

Second molar retention is generally discovered serendipitously, being asymptomatic in $68.5 \%$ of cases $^{3}$. It is thus very rarely a presenting symptom in orthodontics. Precise diagnosis is very difficult to establish, as the numerous etiologies are still not clearly defined. One or several factors may be involved²: germ and alveolar bone, via the dental follicle; gum mucosa, which plays an important role in the last stages of eruption and may induce fibromucosal inclusion; and/or insufficient facial and maxillo-mandibular growth, inducing dentomaxillary dysharmony.
Eruption delay may concern a single tooth, with local etiology; this is the most frequent case ${ }^{25}$. However, a group of teeth or all the teeth may be involved, in which case etiology is more often systemic or genetic ${ }^{16}$.

The present article will list the various etiologies, with precise definitions as needed.

The most useful diagnostic data come from meticulous history-taking and radiology, with possible follicular biopsy. This information, included in a decision tree that will be presented below, guides treatment strategy.
Address for correspondence:

Hélène Guiral-Desnoës

79, Avenue Pablo Picasso 92000 Nanterre,

France helene.guiral@neuf.fr
Article received: 06-05-2015. Accepted for publication: 25-05-2015.

This is an Open Access article distributed under the terms of the Creative Commons Attribution License (http://creativecommons.org/licenses/by/4.0), which permits unrestricted use, distribution, and reproduction in any medium, provided the original work is properly cited. 


\section{LOCAL SECOND MOLAR ERUPTION PATHOLOGY}

A reminder of some definitions will help understanding.

- Impaction: a tooth is said to be impacted when eruption is arrested by a clinically or radiologically detectable obstacle (mechanical failure of eruption: MFE) or by an eruption pathway abnormality (ectopia) ${ }^{21}$. Mesial angulation is often found (fig. 1).

- MFE due to an obstacle in the eruption path, visible on radiography or not $^{24}$, is the main cause of impaction.

\section{Germ abnormalities}

Germ abnormalities impair the communications triggering eruption. They may be hereditary (impaired amelogenesis and dentinogenesis, enamel nodule, etc.) or acquired (trauma).

\section{Dental obstacles}

Loss of space due to unduly early avulsion, absence of guidance by the distal root of the first molar if the lat-

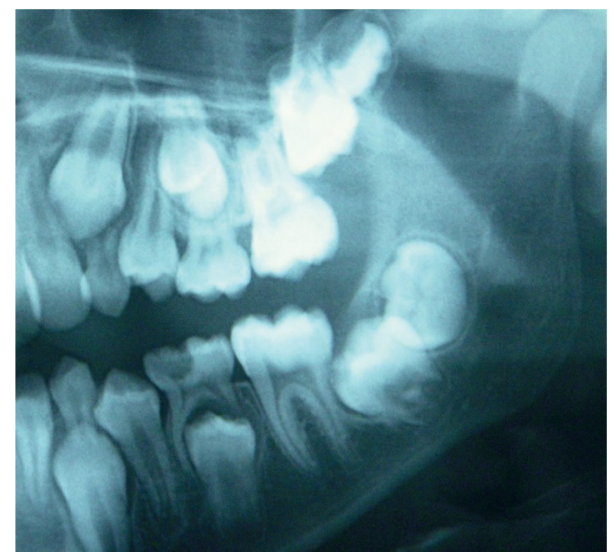

Figure 1

MFE, with impaction of 37 and angulation $>30^{\circ}$. ter has been extracted ("guidance" theory $\left.{ }^{10}\right)$, dentomaxillary dysharmony due to relative macrodontia, short arcade and skeletal growth pattern ${ }^{9}$, as well as supernumerary teeth and/or odontomas may hinder harmonious second molar positioning. Retarded second molar progression may also be due to third molar agenesis (fig. 2).

According to Andreasen, lack of space leads to follicular collision between germs ${ }^{17}$.

\section{Gum obstacles}

Localized gingival hyperplasia.

\section{Tumoral obstacles}

Odontogenic tumor, ameloblastoma, regional odontodysplasia, cyst and myxoma.

Pericoronary hamartoma: certain authors ${ }^{5}$ described a tumor-like tissue deformity composed of an abnormal combination of elements normally

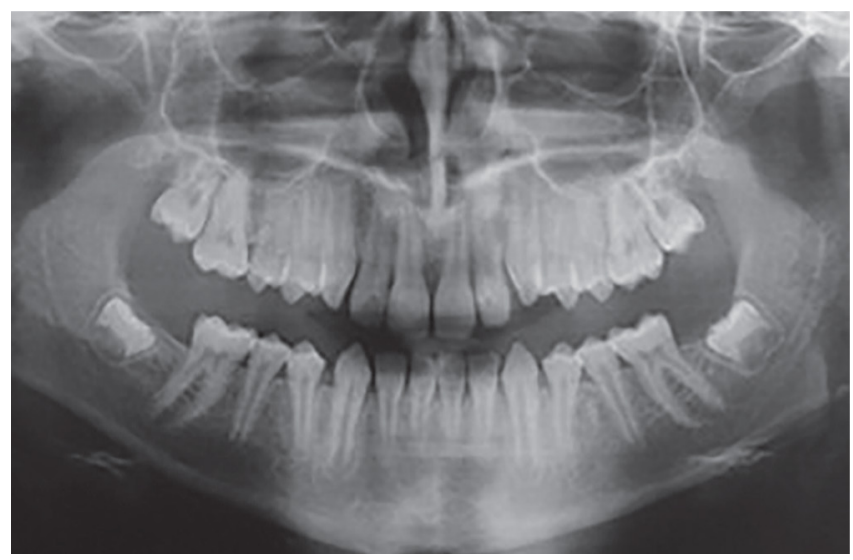

Figure 2

Patient aged 13 years 7 months. Retarded second molar progression with wisdom tooth agenesis. 
found in the organ: osteodentine, cementum, pulp-analog components, multinuclear mesenchymal giant cells, dysplastic dental matrix. The deformity is of embryonic origin, and is also known as dysembryoplasia.

Radiologically, it is characterized by radiolucency surrounding a non-evolved tooth. The constituent elements induce active tissue remodeling, causing gingival fibrosis. Location is most often posterior mandibular, and there is systematically association with a non-erupted tooth. There may or may not be associated syndromes.

This lesion requires particular attention, as the radiologic aspect greatly resembles that of primary failure of eruption (PFE). Differential diagnosis looks for small radioopaque structures within an enlarged image of the follicle. Follicle biopsy analysis confirms diagnosis. In hamartoma, eruption follows its normal course after resection of the fibrous tissue.

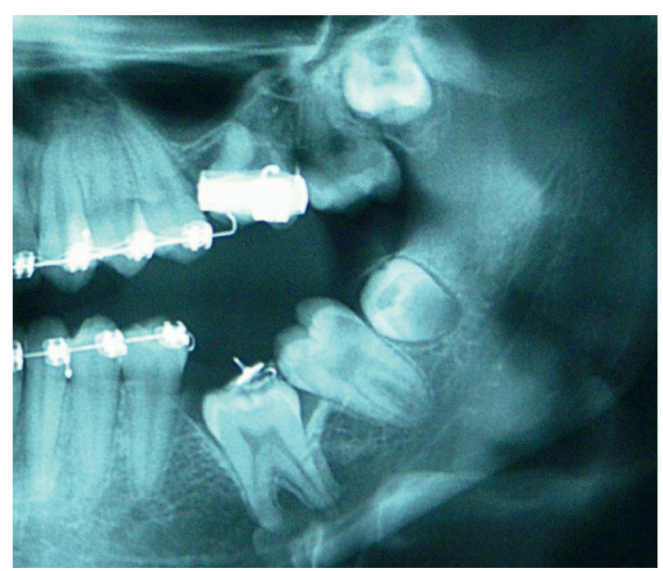

a

\section{Bone obstacles}

Increased local density, or defect in case of cleft $^{23}$.

\section{Mucosal obstacles}

Interference of cheeks, fingers, parafunctions.

\section{Ectopic eruption pathway}

The eruption pathway may be abnormal, usually with medial angulation, causing impaction.

\section{Non-hereditary PFE}

Prevalence ranges from $45 \%$ to $85 \%{ }^{1}$.

PFE (in French, "arrêt idiopathique de l'éruption"6) represents a dissociation between resorption forming the eruption pathway and the various motors of eruption (Proffit and Vig 198120). PFE associates most of these characteristics ${ }^{20}$, but with many phenotypic variants.

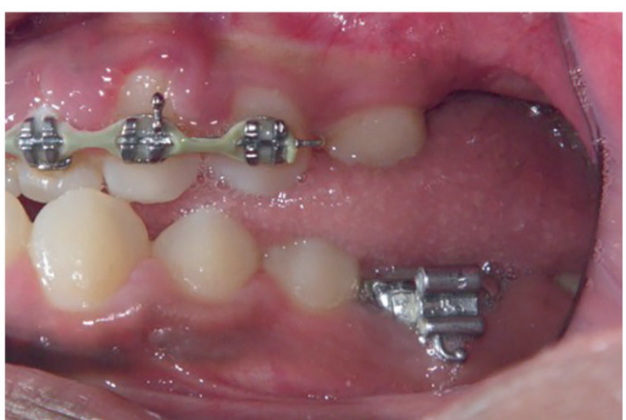

b

Figure 3

a) PFE involving 6, 7 and 8 of quadrants 2 and 3. b) Oral view: posterior infra-occlusion due to PFE. 
Posterior teeth are more involved than anterior teeth. PFE generally begins with the first permanent molar, by ankylosis of the deciduous second molar (fig. 3a). More rarely, premolars and canines may be involved, but never the incisors. Teeth distal to the first affected tooth are all involved to a greater or lesser degree (types I and II).

Affected teeth may have begun eruption but ceased at a certain point: primary and secondary retention. PFE covers a wide phenotypic spectrum, with severity ranging from simple retarded eruption to complete inclusion. Different mutations may be implicated.

Deciduous and permanent teeth may be involved.

The phenomenon may be uni- or bilateral, but is most often asymmetric and unilateral.

The alveolar bone above the crown is resorbed, creating a radiologically visible eruption pathway..

Affected permanent teeth generally show no ankylosis. However, applying orthodontic force to reposition the teeth in the arcade is generally unsuccessful and leads to ankylosis. The affected teeth do not show normal orthodontic response; they may sometimes slowly be pulled for 1 or $2 \mathrm{~mm}$, but then egression stops. Isolated cases (idiopathic, sporadic mutation) have been reported.

The phenomenon induces a posterior gap, the size of which depends on the severity of the pathology (fig. 3b).

When involvement begins only with the second molar, diagnosis cannot be made before 14 years of age; this is known as moderate PFE. Certain wisdom teeth in retention may be concerned by PFE: it is not lack of space that prevents eruption.

Proffit and Vig hypothesized an anteroposterior eruption gradient along the dental lamina, which might explain the greater frequency found in the more posterior teeth $(\mathrm{M} 3++)$.

There may be a genetic link between local molar ankylosis and PFE, as the two may be found in different quadrants in the same subject.

According to Frazier-Bowers ${ }^{12}$, etiology may be distinct between ankylosis, primary retention, secondary retention and PFE.

Idiopathic eruption failure is probably genetic, but etiology may also be multifactorial. Secondary retention, the causes of which remain unelucidated, may associate physiological, mechanical and/or genetic factors ${ }^{12}$. Most cases are related either to ankylosis or, more often, to PFE.

The morphologic characteristics are the same as in hereditary PFE.

\section{SYSTEMIC SECOND MOLAR ERUPTION PATHOLOGIES}

Retention in these cases is usually due to generalized gingival fibrosis, supernumerary teeth or growth defect, rather than
PFE as such. Well-conducted medical history taking regarding patient and forebears guides diagnosis. 


\section{Endocrine etiologies}

Endocrine disorders may affect both craniofacial development and alveolo-dental growth; eruption may be affected to a greater or lesser degree according to the hormone in question and age at onset. Genetic and acquired endocrine disorders may be distinguished. Bone base and alveolar process growth may be involved, greatly delaying eruption by slowing alveolar bone formation or causing MFE by dentomaxillary dysharmony.

Hypothalamic and pituitary functions, and especially the $\mathrm{GHRH}$ GH-IGF-1 axis, are determining in regulating growth ${ }^{4}$. Acting via IGF-1, growth hormone $(\mathrm{GH})$ or somatotropin is the main growth determinant (hypopituitarism), and is synthesized and released by the anterior pituitary. Thyroid hormones also play an essential role in statural growth, and especially in the skeletal maturation of the growth plate (hypothyroidism, hypoparathyroidism). Thyroxin stimulates the growth cartilage, and thyrocalcitonin inhibits bone catabolism and promotes osteogenesis. The effects are both direct and indirect, via activation of $\mathrm{GH}$ gene transcription. PTH (parathyroid hormone) has hypercalcemic and hypophosphatemic action, stimulating both bone formation and resorption, thus renewing the bone while maintaining skeletal integrity. $\mathrm{PTHrP}$ (parathyroid-related protein) is required for dental eruption (Philbrick, cited by Frazier-Bowers, Puranik, Mahaney ${ }^{12}$ ). The gonadic steroids LH (luteinizing hormone) and FSH (follicle-stimulating hormone) are mainly involved in the acceleration of growth observed at puberty (hypogonadism).
Moreover, developmental and hormonal genes interact: gene promoter sequences show hormonal response.

Local paracrine regulation and circulating hormones control bone tissue development and remodeling throughout life.

\section{Deficiencies}

- Vitamin D deficiency (rickets): One of the three main actions of vitamin $D$ is on bone tissue, itself involved in craniofacial edification. Vitamin D is a steroid that can be seen both as a hormone and as a vitamin. It is a major element in phosphocalcium metabolism. 1,25 $(\mathrm{OH}) 2 \mathrm{D}$ shows hypercalcemic and hyperphosphatemic action on both bone and teeth ${ }^{4}$.

- Vitamin A or C deficiency induces gingival hyperplasia ${ }^{24}$, and may also delay eruption.

- Likewise, retarded eruption is found in severe prematurity ${ }^{16}$.

\section{Drug-related etiologies}

Many medical treatments may slow eruption: long-course chemotherapy (fig. 4), or prostaglandin pathway blockers that reduce periodontal osteoclastic activity: aspirin, acetaminophen, ibuprofen, indomethacin, clodronate $^{26}$, dihydantoin (used to treat epilepsy), or phenytoin (causing gingival hyperplasia) ${ }^{24}$. Chemically modified tetracyclines inhibit matrix metalloproteinase. Bisphosphonates (pamidronate and alendronate), prescribed to children for primary and secondary osteoporosis, including impaired osteogenesis, delay or inhibit dental eruption by impairing activity $^{14}$. 


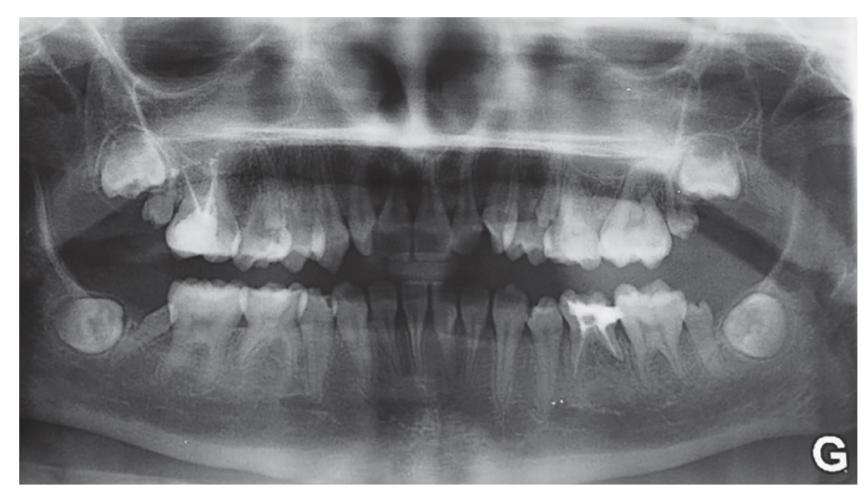

Figure 4

Second molar abnormality and retarded eruption secondary to chemotherapy during infancy.

\section{Genetic etiologies}

\section{Genetic etiologies associated with syndromes}

Many syndromes are associated with retarded eruption of the permanent teeth (fig. 5).

In 2002, Wise listed 25, half of which involved genetic mutation ${ }^{27}$. Such syndromes are not systematically familial, and may implicate sporadic mutation. Except for Levy-Hollister syndrome and progeria, which affect only permanent teeth, all other syndromes affect both dentitions, usually inducing severe delay rather than primary failure of eruption. eruption may be impacted at different stages, with correspondingly different severity.

The only syndromes presently known to be associated with true PFE are:

- Albers-Schönberg osteopetrosis;

- GAPO syndrome (Anderson and

Pindborg: OMIM \#230740);

- and Fairbanks syndrome (osteoglophonic dysplasia).

Early diagnosis of dental abnormalities $^{19}$ is useful, and indispensable to broader genetic diagnosis and ap- propriate treatment, with possibility of genetic counseling for patient and family. Drawing up an individualized orodental prevention program, and treatment sequence planning to conserve existing dental potential, improve esthetics and function and conserve dental stock until adulthood (fig; 6).

Non-syndromic hereditary PFE In $15-45 \%$ of cases 1 , PFE may be familial.

The exact etiology of PFE remains undetermined, but reports of hereditary cases $8,13,21,28$ tend to show a link with a genetic mutation of incomplete penetrance and variable expression. Dominant transmission linked to the $\mathrm{X}$ chromosome cannot be ruled out. Frazier-Bowers' team situates the main origin of PFE in the alveolar bone ${ }^{12}$. Candidate genes are thus those involved in bone remodeling. It may be wondered whether PFE and ankylosis belong to the same spectrum of mutations ...

In humans, PFE has been associated with mutations in Runx2 (RUNrelated transcription factor 2), TRAF 6 (TNF Receptor Associated Factor 6), and FGFR1-3 (Fibroblast Growth Factor Receptor).

Recently, PTH1R mutations were identified in cases of non-syndromic familial PFE with autosomal dominant high-penetrance transmission and variable expression ${ }^{8,13}$. Haploinsufficiency in the common PTH1R receptor of $\mathrm{PTH}$ and $\mathrm{PTHrP}$ is probably implicated in PFE.

Non-syndromic PFE is now the $5^{\text {th }}$ most frequent pathology linked to PTH1R mutation, after Blomstrand's syndrome (chondrodysplasia), Ollier's disease (enchondromatosis), Eiken's 
Figure 5

Table of syndromes associated with retarded and/or arrested eruption, according to Wise ${ }^{27}$, completed by Molla ${ }^{15}$, Suri24 and Orphanet ${ }^{18}$.

\begin{tabular}{|c|c|c|c|c|}
\hline Syndrome & Eruption phenotype & Genetic defect & Transmission & Ref. OMIM \\
\hline $\begin{array}{l}\text { Cleidocranial dysos- } \\
\text { tosis: Pierre Marie } \\
\text { and Sainton }\end{array}$ & $\begin{array}{c}\text { Generalized retarda- } \\
\text { tion by lack of cel- } \\
\text { lular cementum }\end{array}$ & $\begin{array}{l}6 \text { mutations } \neq \text { of } \\
\text { PEBP } 2 \alpha A / C B F A 1\end{array}$ & Autosomal dominant & \# 119600 \\
\hline $\begin{array}{c}\text { Albers-Schoenberg } \\
\text { osteopetrosis }\end{array}$ & Idiopathic arrest & TRAF6 & $\begin{array}{c}\text { Autosomal dominant } \\
\text { or recessive }\end{array}$ & $\begin{array}{c}\text { \#259700, \#259710, } \\
\text { \#259730 }\end{array}$ \\
\hline $\begin{array}{l}\text { GAPO (Anderson } \\
\text { and Pindborg) }\end{array}$ & $\begin{array}{l}\text { Idiopathic arrest, } \\
\text { retarded growth }\end{array}$ & & Autosomal recessive & \#230740 \\
\hline $\begin{array}{c}\text { Osteopathia striata } \\
\text { with cranial sclerosis }\end{array}$ & Idiopathic arrest & $X q 11,2$ & Autosomal dominant & \# 300373 \\
\hline $\begin{array}{c}\text { Fairbanks' osteoglo- } \\
\text { phonic dysplasia }\end{array}$ & $\begin{array}{l}\text { Idiopathic arrest of } \\
\text { permanent teeth }\end{array}$ & $8 p 11,23 ; p 11,22$ & Autosomal dominant & \# 166250 \\
\hline Singleton-Merten & $\begin{array}{l}\text { Dental dysplasia, } \\
\text { permanent tooth } \\
\text { retardation }\end{array}$ & & $\begin{array}{l}\text { Autosomal } \\
\text { dominant? }\end{array}$ & \# 182250 \\
\hline Aarskog & Retardation & $\begin{array}{l}\text { FGDY1 coding } \\
\text { growth reg. fact. }\end{array}$ & $\begin{array}{l}\text { Linked to } X \\
\text { recessive }\end{array}$ & \#100050 \\
\hline Acrodysostosis & $23 \%$ retardation & & Autosomal dominant & \#248400? \\
\hline \begin{tabular}{|c|} 
Albright hereditary \\
osteodystrophy: \\
pseudohypoparathy- \\
roidism \\
\end{tabular} & Retardation & $\begin{array}{l}\text { mutations } \neq \text { GNAS1 } \\
\text { (protein G) 20q13,32 }\end{array}$ & Autosomal dominant & \#174800 \\
\hline Cherubism & $\begin{array}{c}\text { Retardation by bone } \\
\text { obstacle, tumor, } \\
\text { MFE }\end{array}$ & $\begin{array}{c}\text { Mutation SH3BP2 } \\
\text { gene (4p16.3.) }\end{array}$ & $A D$ & \#118400 \\
\hline Noonan & Cherubism, MFE & $\begin{array}{l}\text { PTPN11 (chr 12) } \\
\text { KRAS, SOS1, and } \\
\text { RAF1 genes }\end{array}$ & $A D$ & \#163950 \\
\hline Ramon & $\begin{array}{c}\text { Cherubism, MFE + } \\
\text { gingival fibromatosis }\end{array}$ & $?$ & AR & \#266270 \\
\hline $\begin{array}{l}\text { Recklinghausen's } \\
\text { disease: Neurofi- } \\
\text { bromatosis } 1\end{array}$ & Cherubism, MFE & NF1 gene (17q11.2) & $A D$ & \#162200 \\
\hline $\begin{array}{c}\text { Apert } \\
\text { acrocephalosyn- } \\
\text { dactily }\end{array}$ & $\begin{array}{c}\text { Generalized re- } \\
\text { tardation ectopia, } \\
\text { hypodontia }\end{array}$ & $\begin{array}{c}\text { Mutation FGFR2 } \\
\text { gene } 10 q 26,13\end{array}$ & Autosomal dominant & $\begin{array}{l}\# 101200 \\
\text { IV \#201020 }\end{array}$ \\
\hline $\begin{array}{l}\text { Carpenter acroceph- } \\
\text { alosyndactily }\end{array}$ & $\begin{array}{l}\text { (1) bone density hin- } \\
\text { dering resorption }\end{array}$ & $\begin{array}{l}\text { MEGF8, RAB23 } \\
6 p 11,2 / 19 q 13,2\end{array}$ & $A R$ & $\begin{array}{l}\# 201000 \\
\# 614976\end{array}$ \\
\hline
\end{tabular}




\begin{tabular}{|c|c|c|c|c|}
\hline Syndrome & Eruption phenotype & Genetic defect & Transmission & Ref. OMIM \\
\hline $\begin{array}{c}\text { Ellis van Crevelt } \\
\text { Chondroectodermal } \\
\text { dysplasia }\end{array}$ & $\begin{array}{l}\text { Retardation, oligo- } \\
\text { dontia }\end{array}$ & $\begin{array}{l}\text { 4p16 (EVC1) } \\
4 \text { p16 (EVC2) }\end{array}$ & $\begin{array}{c}\text { Autosomal recessive } \\
\text { limbine }\end{array}$ & XLHED \#305100 \\
\hline Cockayne & Retardation & CSB gene (ERCC6) & Autosomal recessive & \\
\hline De Lange & Retardation & SHOT ??? & $\begin{array}{c}\text { Autosomal } \\
\text { dominant??? }\end{array}$ & \\
\hline Dubowitz & $\begin{array}{l}\text { Retardation, hypo- } \\
\text { dontia, short stature }\end{array}$ & $?$ & $\begin{array}{l}\text { Autosomal } \\
\text { recessive?? }\end{array}$ & \#223370 \\
\hline $\begin{array}{c}\text { Gorlin Cohen, } \\
\text { Chaudry-Moss } \\
\text { Frontometaphyseal } \\
\text { dysplasia }\end{array}$ & $\begin{array}{l}\text { Retardation, decidu- } \\
\text { ous ankylosis }\end{array}$ & $?$ & $\begin{array}{l}\text { Autosomal domi- } \\
\text { nant??? Linked to } \\
\quad \text { X??? }\end{array}$ & \\
\hline $\begin{array}{c}\text { Goltz } \\
\begin{array}{c}\text { Focal dermal hypo- } \\
\text { plasia }\end{array} \\
\end{array}$ & $\begin{array}{c}\text { Retardation, } \\
\text { hypodontia, dental } \\
\text { hypoplasia }\end{array}$ & Xp11,23 & $\begin{array}{c}\text { Linked to } X, \\
\text { dominant, fatal in } \\
\text { hemizygotic boys }\end{array}$ & \#305600 \\
\hline $\begin{array}{l}\text { Hunter Mucopoly- } \\
\text { saccharoidosis II }\end{array}$ & Retardation & $\begin{array}{c}\text { Mutations } \neq \text { IDS } \\
\text { gene } X q 28\end{array}$ & Linked to $X$ & \#309900 \\
\hline $\begin{array}{l}\text { Bloch-Sulzberger } \\
\text { Incontinentia pig- } \\
\text { menta }\end{array}$ & $\begin{array}{c}\text { Retardation, hypo- } \\
\text { dontia in } 80 \% \text { of } \\
\text { cases }\end{array}$ & $\begin{array}{l}\text { Xp11.2, rarely IKK } \\
\text { gene Xq28 }\end{array}$ & $\begin{array}{l}\text { Linked to } X \text {, domi- } \\
\text { nant, fatal in boys }\end{array}$ & \#308300 \\
\hline $\begin{array}{l}\text { Killian/Teschler-Nico- } \\
\text { la Pallister Killian }\end{array}$ & Retardation & $\begin{array}{c}\text { Tetrasomia } 12 p \text { in } \\
\text { mosaic }\end{array}$ & $\begin{array}{l}\text { Chromosomal } \\
\text { abnormality }\end{array}$ & PKS \#601803 \\
\hline Levy- Hollister & $\begin{array}{c}\text { Deciduous retarda- } \\
\text { tion }\end{array}$ & $?$ & Autosomal dominant & \\
\hline $\begin{array}{c}\text { Maroteaux-Lamy } \\
\text { Mucopolysaccharoi- } \\
\text { dosis type } \mathrm{VI}\end{array}$ & $\begin{array}{l}\text { Retardation, micro- } \\
\text { dontia }\end{array}$ & $\begin{array}{c}\text { Mutations } \neq \text { ASB } \\
\text { gene } 5 q 14,1\end{array}$ & Autosomal recessive & MPS6\#253200 \\
\hline $\begin{array}{l}\text { Hurler's disease } \\
\text { Mucopolysaccharoi- } \\
\text { dosis type I }\end{array}$ & Retardation & $\begin{array}{l}\text { IDUA - Iduronidase, } \\
\text { alpha-L 4p16,3? }\end{array}$ & $A R$ & \#607014 \\
\hline $\begin{array}{c}\text { Trichorhinophalan- } \\
\text { geal syndrome type } \\
1 \text { or } 3\end{array}$ & Retardation & $\begin{array}{c}\text { Mutations } \\
\text { TPRS1 gene local- } \\
\text { ized in } 8 q 23,3\end{array}$ & $A D$ & \#190350 \\
\hline $\begin{array}{c}\text { Rothmund-Thomson } \\
\text { Congenital poikilo- } \\
\text { derma }\end{array}$ & $\begin{array}{l}\text { Retardation, agen- } \\
\text { esis,. supernum., } \\
\text { hypgonadism }\end{array}$ & $8 q 24,3$ & & \#268400 \\
\hline $\begin{array}{c}\text { Papillon-Léage } \\
\text { Orodigitofacial syn- } \\
\text { drome I }\end{array}$ & Retardation & $\begin{array}{l}\text { Mutation OFD1 } \\
\text { gene }\end{array}$ & & \#311200 \\
\hline $\begin{array}{c}\text { Mohr Orodigitofacial } \\
\text { syndrome II }\end{array}$ & Retardation & & & \#252100 \\
\hline $\begin{array}{l}\text { Hyperimmuno } \\
\text { globulinemia } \mathrm{E}\end{array}$ & $\begin{array}{l}\text { Retardation despite } \\
\text { resorption }\end{array}$ & & Autosomal dominant & \\
\hline
\end{tabular}




\begin{tabular}{|c|c|c|c|c|}
\hline Syndrome & Eruption phenotype & Genetic defect & Transmission & Ref. OMIM \\
\hline $\begin{array}{l}\text { Osteogenesis im- } \\
\text { perfecta Type I }\end{array}$ & $\begin{array}{l}\text { Retardation, dental } \\
\text { dysplasia }\end{array}$ & $\begin{array}{l}\text { COL1A1 and } \\
\text { COL1A2 }\end{array}$ & $\begin{array}{c}\text { Autosomal } \\
\text { dominant, variable } \\
\text { expression }\end{array}$ & \#259410? \\
\hline $\begin{array}{l}\text { Hutchinson- Gilford } \\
\text { progeria }\end{array}$ & $\begin{array}{c}\begin{array}{c}\text { Retardation of both } \\
\text { dentitions, rare hy- } \\
\text { podontia permanent } \\
\text { anodontia }\end{array} \\
\end{array}$ & $\begin{array}{l}\text { ADN helicase, } \\
\text { telomerase }\end{array}$ & Autosomal recessive & \#176670 \\
\hline Pyknodysostosis & $\begin{array}{c}\text { Retardation, } \otimes \text { bone } \\
\text { density hindering re- } \\
\text { sorption, sometimes } \\
\text { anodontia }\end{array}$ & Cathepsin K gene & Autosomal recessive & \\
\hline Rothmund-Thomson & $\begin{array}{l}\text { Retardation, micro- } \\
\text { dontia, supernumer- } \\
\text { ary or missing teeth. }\end{array}$ & $\begin{array}{c}\text { 8q24.3 RECQLA, } \\
\text { WRN, BLM }\end{array}$ & Autosomal recessive & \#268400 \\
\hline Rubinstein-Taybi & $\begin{array}{l}\text { Retardation, hypo- } \\
\text { dontia }\end{array}$ & $\begin{array}{l}\text { 16p13 CREBBP, } \\
22 q 13.2 \text { EP300 }\end{array}$ & Autosomal dominant & $\begin{array}{l}\text { RSTS1\#180849 } \\
\text { RSTS2\#613684 }\end{array}$ \\
\hline $\begin{array}{l}\text { Oculofaciocardio- } \\
\text { dental syndrome }\end{array}$ & Retardation & $\begin{array}{c}\text { Xp11.4 (BCOR) BL6 } \\
\text { corepressor }\end{array}$ & $\begin{array}{l}\text { Linked to } X \text {, domi- } \\
\text { nant, fatal in boys }\end{array}$ & $\begin{array}{l}\text { BCOR \#300485 } \\
\text { MCOPS2\#300166 }\end{array}$ \\
\hline $\begin{array}{c}\text { Cerebro-oculo-den- } \\
\text { to-auriculo-skeletal } \\
\text { syndrome } \\
\end{array}$ & $\begin{array}{l}\text { Retardation, cuspid } \\
\text { form anomalies }\end{array}$ & $\begin{array}{c}\text { Collagen gene } \\
\text { defect? }\end{array}$ & Autosomal recessive & \#600373 \\
\hline Down's & Retardation & $21 q 22,13$ & $\begin{array}{l}\text { Chromosomal } \\
\text { abnormality }\end{array}$ & $\begin{array}{l}\text { DSCR3\#605298 } \\
\text { DSCR4\#604829 } \\
\text { DSCR6\#609892 } \\
\end{array}$ \\
\hline Turner MRXST & Retardation & Xp11,22 & & \#300706 \\
\hline $\begin{array}{c}\text { Gardner } \\
\text { Familial adeno- } \\
\text { matous polyposis } \\
\text { (FAP)1 }\end{array}$ & $\begin{array}{c}\text { Generalized retarda- } \\
\text { tion, ankylosis } \\
\text { by cell proliferation, } \\
\text { tumors } \\
\end{array}$ & APC gene $5 q 22,2$ & $?$ & \#175100 \\
\hline Gorlin NBC BCNS & $\begin{array}{l}\text { MFE, odontogenic } \\
\text { keratocysts }\end{array}$ & $\begin{array}{l}\text { Mutation } \mathrm{PTCH} 1 \\
\text { gene }\end{array}$ & $A D$ & \#109400 \\
\hline $\begin{array}{c}\text { (Axenfeld)Rieger } \\
1,2,3\end{array}$ & $\begin{array}{c}\text { Retardation?, oligo- } \\
\text { dontia } \\
\text { 凶size }\end{array}$ & $\begin{array}{c}\text { PITX2 (4q25) and } \\
\text { FOXC1 genes }(6 p 25)\end{array}$ & $\begin{array}{c}\text { Autosomal } \\
\text { dominant, strong } \\
\text { penetrance }\end{array}$ & $\begin{array}{c}\# 601542 \\
\text { RIEG1 \#180500 } \\
\text { RIEG2\#601499 } \\
\text { RIEG3\#602482 } \\
\end{array}$ \\
\hline $\begin{array}{c}\text { Parry Romberg } \\
\text { Progressive hemifa- } \\
\text { cial atrophy }\end{array}$ & $\begin{array}{l}\text { Retardation by bone } \\
\text { obstacle }\end{array}$ & $?$ & $A D$ & \#141300 \\
\hline Lowry-MacLean & Retarded growth & $?$ & $A D$ & \#600252 \\
\hline $\begin{array}{l}\text { Murray-Puretic- } \\
\text { Drescher } \\
\text { Juvenile hyaline } \\
\text { fibromatosis } \\
\end{array}$ & $\begin{array}{c}\text { Gingival fibroma- } \\
\text { tosis }\end{array}$ & $\begin{array}{l}\text { 4q21 CMG2 } \\
\text { (capillary morpho- } \\
\text { genesis protein 2) }\end{array}$ & $A R$ & \#228600 \\
\hline $\begin{array}{c}\text { Rutherfurd } \\
\text { Oculodental syn- } \\
\text { drome }\end{array}$ & $\begin{array}{l}\text { Gingival fibroma- } \\
\text { tosis }\end{array}$ & $?$ & $A D$ & \#180900 \\
\hline
\end{tabular}




\begin{tabular}{|c|c|c|c|c|}
\hline Syndrome & Eruption phenotype & Genetic defect & Transmission & Ref. OMIM \\
\hline Cross & $\begin{array}{c}\text { Gingival fibroma- } \\
\text { tosis }\end{array}$ & $?$ & $?$ & \\
\hline $\begin{array}{l}\text { Zimmermann } \\
\text { Laband }\end{array}$ & $\begin{array}{c}\text { Gingival fibroma- } \\
\text { tosis }\end{array}$ & $\begin{array}{c}\text { critical region in } \\
3 p 14.3 ?\end{array}$ & $A D ?$ & $\# 135500$ \\
\hline $\begin{array}{c}\text { Aspartylglycosami- } \\
\text { nuria (AGU) }\end{array}$ & ??? & $\begin{array}{c}\text { 14q32-q33 Finland } \\
\text { mutations AGUfin } \\
4 q 34,3\end{array}$ & $A R$ & \#208400 \\
\hline $\begin{array}{l}\text { Hereditary amelio- } \\
\text { genesis imperfecta }\end{array}$ & $\begin{array}{l}\text { Retardation, mor- } \\
\text { phologic anomalies }\end{array}$ & Mutations $\neq$ gene & $\begin{array}{l}\neq \text { transmission } \\
\text { modes }\end{array}$ & 14 formes \\
\hline PFE & $\begin{array}{l}\text { Complete or partial } \\
\text { idiopathic arrest }\end{array}$ & $\begin{array}{c}3 \text { mutations PTHR1 } \\
\text { (13) 3p24.3-p14.3 ; } \\
3 p 21,31\end{array}$ & $A D$, non-syndromic & \#125350 \\
\hline
\end{tabular}

syndrome and Murk-Jansen metaphyseal chondrodysplasia. In general, however, PFE patients do not have associated skeletal abnormalities. However, Frazier-Bowers' team recently reported 2 families with associated osteoarthritis $^{11}$. It thus seems unlikely that PTH1R haplo-insufficiency prevents systemic osteoclast formation and functioning. Non-syndromic PFE is probably caused by defective interaction in epithelial-mesenchymal cells immediately adjacent to the eruption path, altering the delicate balance between bone resorption and formation $^{8}$. In PFE, osteoclasts seem not to express functional PTH1R receptors on their surface. Instead, the dental follicle may contain a paracrine or juxtacrine signaling pathway comprising PTH1R positive cells on the surface. Moreover, the fact that only posterior regions are involved remains unexplained. PTH1R gene products may have specific temporal and spatial action $^{13}$.

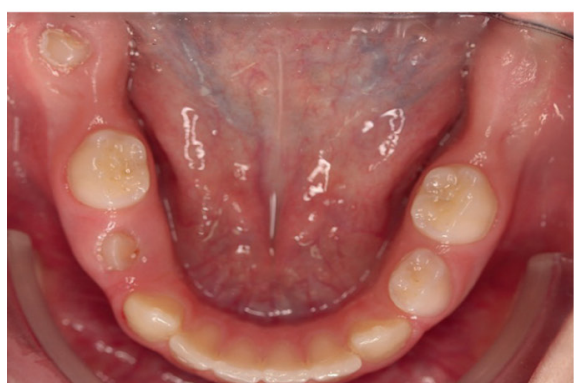

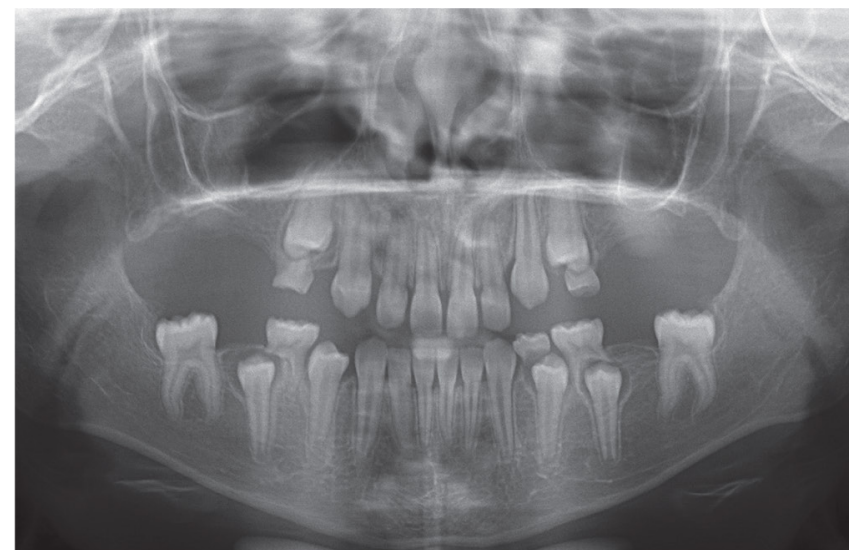

b

Figure 6

a) Agenesis and retarded eruption; 2 other siblings, father and grandfather affected; syndrome nonidentified. b) Panoramic view, 13 years. 
Although PTH1R gene mutations seem clearly implicated in PFE, other genes may also well be involved. PTH1R mutation affects a whole local paracrine regulation system in PFE, although this remains to be identified.

In 2011, Yamagushi's team ${ }^{28}$ identified 3 other PTH1R gene mutations that may underlie PFE $(6$ mutations in all so far?), as well as mutations of the TGFBR2 and PROKR2 genes involved in bone metabolism.

\section{Other systemic etiologies 5}

\section{Ionizing radiation}

Accidental uranium irradiation or head and neck tumor radiotherapy may cause ankylosis, periodontal ligament lesions and maxillary growth defect.

\section{Systemic diseases}

HIV? Anemia, kidney failure...

\section{Drugs}

Nicotine, other drugs...
Administrative information:

Family name:

Given name:

Date and place of birth:

Address/country:

Reason for consultation:

General health status :

Was your child full-term?

Were there complications during pregnancy? Were medical treatments prescribed? If so, which? For how long?

Did your child have vitamin deficiencies? Feeding problems? Does he/she take vitamin D supplements every winter?

Does your child take medicines? If so, which? For how long?

Is your child's growth retarded? Late puberty? Does he/she take treatment for that? If so, which? For how long?

Does your child have epilepsy? Hearing problems?

Has your child had radiation therapy for head and neck tumor?

Has your child lived close by a nuclear accident?
Family and medical history:

Have you consulted a geneticist?

Do you have family members with bone disease? Cartilage disease?

Do you have family members with dental abnormalities: eruption problems? Agenesis? Abnormal tooth shape?

Dental history:

Has your child recently had molar region pain?

How long did it last?

Were there associated swellings or discharge?

When did your child produce the first milk tooth?

When did your child lose the first milk tooth? (mandibular incisor)

Did all the milk teeth fall out naturally, without needing extraction?

Has your child had dental treatment?

If so, what? (Prevention/restoration/extraction?)

Has your child sustained facial trauma? Mandibular fracture? Maxillary fracture?

Has your child had orthodontic treatment?

If so, what type? Please send initial documents if possible. 


\section{QUESTIONNAIRE}

Based on the Orphanet questionnaire (fig. 7), a certain number of points were raised to guide history-taking toward the various above etiologies. The questionnaire should remain open-ended, with new observations being added from new cases.

\section{DIAGNOSTIC AND THERAPEUTIC DECISION-TREES}

Depending on the answers to the history-taking questionnaire, clinical observation and radiographic examinations, various authors, 722,24 have published therapeutic decisiontrees. These have been recast in the light of recent discoveries (figs 8 and 9).

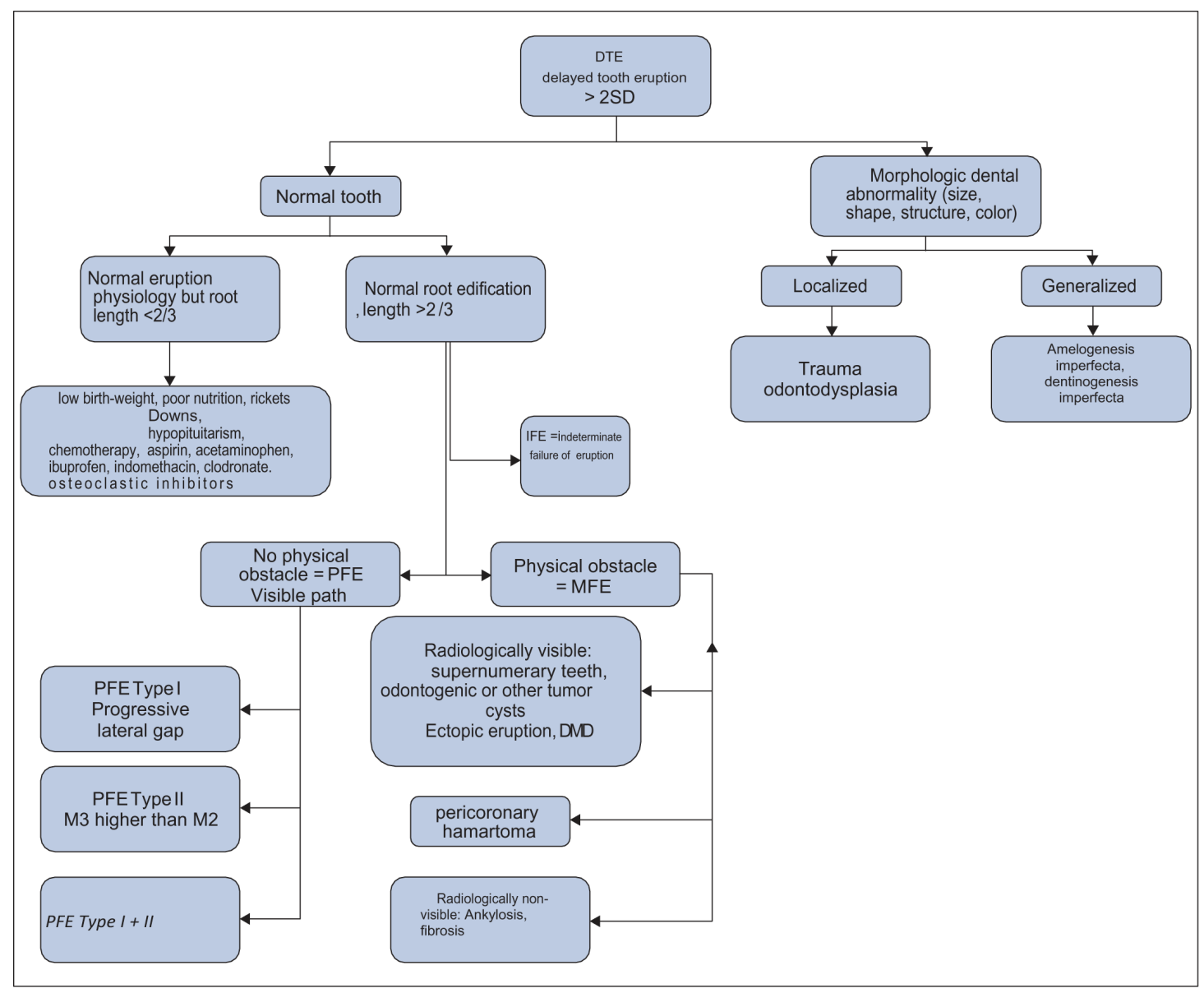

Figure 8

Differential diagnosis and therapeutic decision tree ${ }^{22}$, Suri2 ${ }^{24}$ 


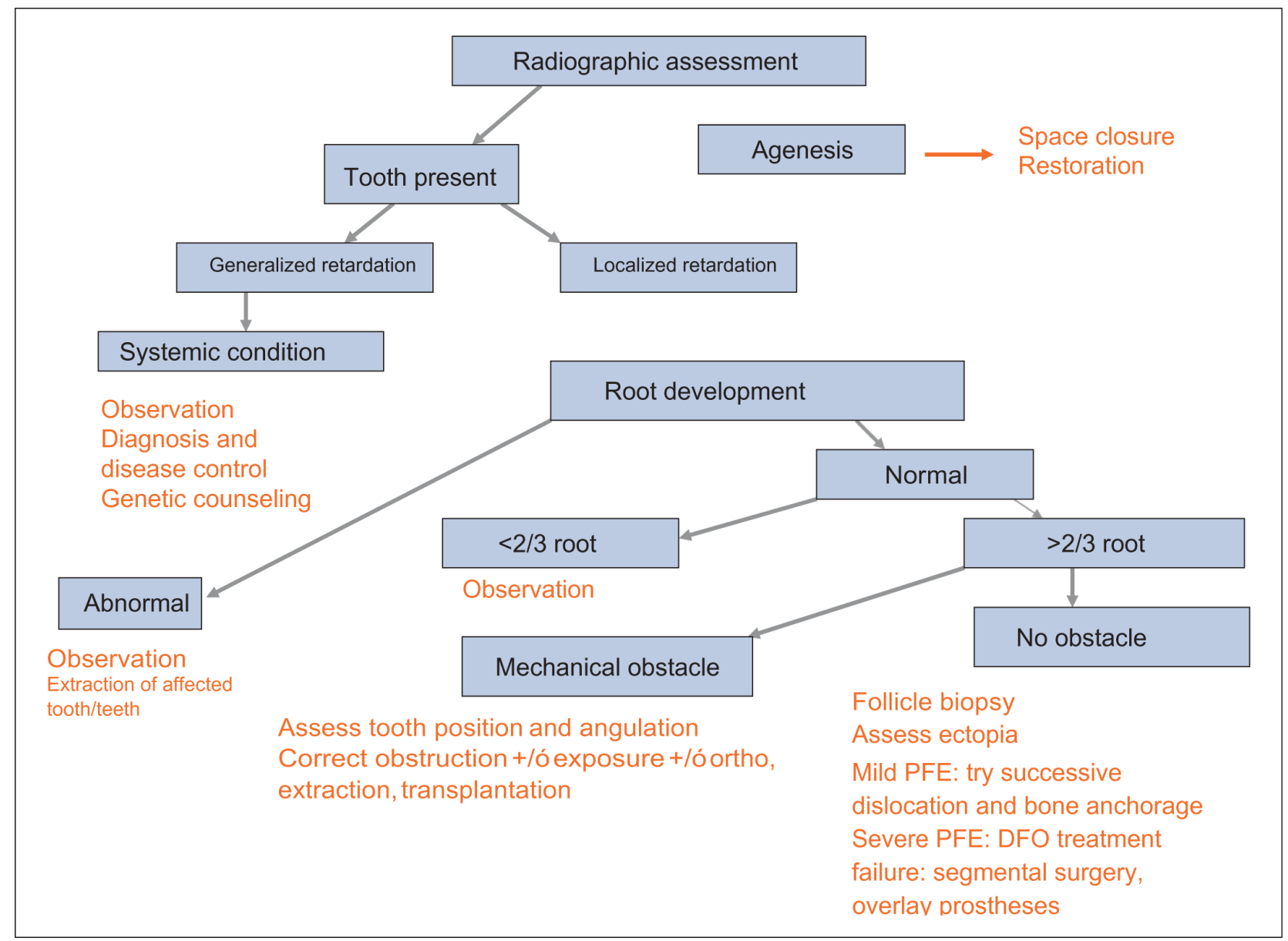

Figure 9

Therapeutic decision tree, Castaneda?

\section{CONCLUSION}

This long list of possible local or systemic etiologies in case of retarded second molar development allows optimization of treatment.

The mechanism of PFE and the role of osteoclasts remain unknown: elucidation would allow prevention and early treatment. Too often, it is the tooth's response to orthodontic efforts that points to retrospective diagnosis. A tooth that fails to get positioned despite adapted treatment is very likely affected by PFE. It is preferable to have established diagnosis ahead of any mechanical intervention, so as to be able to warn the patient of the uncertainty of outcome and to plan bone anchorages. Diagnosis should be precise, informative and evidence-based. It requires 
knowledge of orthodontics, developmental biology and genetics. It may come serendipitously during routine consultation with the dental surgeon, or in the light of a syndromic presentation. The dental surgeon should be able to make such a diagnosis or refer patient and family to a specialist con- sultation. The diagnostic procedure places the patient in the center of the project, and governs both clinical management and the development of research programs ${ }^{19}$.

Conflict of interest

The author declares no conflicts of interest.

\section{REFERENCES}

1. Ahmad S, Bister D, Cobourne MT. The clinical features and aetiological basis of primary eruption failure. Eur J Orthod 2006;28(6):535-540.

2. Berberi $A$, Le Breton $G$, Womant $H$. Deuxième molaire mandibulaire incluse : à propos d'un cas. Revue d'Odonto-Stomatologie 1993;22(3):245-251.

3. Bereket C, Cakir-Özkan N, Sener I, Kara I, Aktan A-M, Arici N. Retrospective analysis of impacted first and second permanent molars in the Turkish population: a multicenter study. Med Oral Pathol Oral Cir Bucal 2011;16(7):e874-8.

4. Besson A, Menuelle P, Ferri J, Berdal A. Endocrinopathies et dysmorphoses craniofaciales : intérêt pour l'orthodontiste. International Orthodontics 2006;4:229-240.

5. Buloto Schmidt L, Bravo-Calderon DM, Teixeira Soares C, Tostes Oliveira D. Hyperplastic Dental Follicle: a case report and Literature Review. Case Reports in Dentistry 2014;ID 251892, 7 p.

6. Canutj A, Ganda JL. Éruption incomplète des molaires permanentes : étude de 22 cas. Rev Orthop Dento-Faciale 1994;28:261-277.

7. Castaneda B. Analyse du rôle des Ostéoclastes dans la croissance dento-alvéolaire in vivo: effets de la surexpression ciblée de RANK dans le lignage ostéoclastique chez des souris CD1 sauvages et mutées pour msx2. Thèse de $3^{e}$ cycle.

8. Decker $\mathrm{E}$, et al. PTHR1 loss-of-function mutations in familial, nonsyndromic primary failure of tooth eruption. Am J Hum Genet 2008;83(6):781-786.

9. Desnoës H. Anomalies d'évolution des deuxièmes molaires et traitement orthodontique sans extractions de prémolaires. Gestion de l'encombrement postérieur. Rev Orthop Dento-Faciale 2014;48:279-290.

10. Ferro F, Funiciello G, Perillo L, Chiodini P. Mandibular lip bumper treatment and second molar eruption disturbances. Am J Orthod Dentofacial Orthop 2011;139(5):622-627.

11. Frazier-Bowers SA, et al. Novel Mutations in PTH1R Associated with primary failure of eruption and osteo- arthritis. J Dent Res 2014;93(2):134-139.

12. Frazier-Bowers SA, Puranik CP, Mahaney MC. The etiology of eruption disordersfurther evidence of a "genetic paradigm". Semin Orthod 16(3):180-185.

13. Frazier SA, Simmons D, Wright T, Proffit WR, Ackerman JL. Primary failure of eruption and PTH1R: the importance of a genetic diagnosis for orthodontic treatment planning. Am J Orthod Dentofacial Orthop 2010;137(2):160.e1-160.e7.

14. Hiraga T, Ninomiya T, Hosoya A, Nakamura H. Administration of the biphosphonate zoledronic acid during tooth development inhibits tooth eruption and formation and induces dental abnormalities in rats. Calcif Tissue Int 2010;86(6):502-510.

15. Molla M, et al. Odontogénétique. EMC 2006;22-001- A-05. 
16. Moulis E, Favre de Thierrens C, Goldsmith MC, Torres JH. Anomalies de I'éruption EMC 2003;4-014- C-60.

17. Nagpal A, Sharma G, Sakar A, Pai KM. Eruption disturbances: an aetiologicalcum-management perspective. Dentomaxillofac Radiol 2005;34(1):59-63.

18. Orphanet http://www.orpha.net.

19. Phenodent http://www.phenodent.org/.

20. Proffit WR, Vig KWL. Primary failure of eruption: a possible cause of posterior open bite. Am J Orthod 1981;80(2):173-190.

21. Raghoebar GM, Ten Kate LP, Hazenberg CAM, Boering G, Vissink A. Secondary retention of permanent molars: a report of five families. J Dent 1992;20:277-282.

22. Sivakumar A, Valiathan A, Gandhi S, Mohandas AA. Idiopathic failure of eruption of multiple permanent teeth: report of 2 adults with a highlight on molecular biology. Am J Orthod Dentofacial Orthop 2007;132(5):687-692.

23. Stellzig-Eisenhauer $A$, et al. Primary failure of eruption (PFE)- Clinical and molecular genetics analysis. J Orofac Orthop 2010;71:6-16.

24. Suri L, Gagari E, Vastardis H. Delayed tooth eruption: pathogenesis, diagnosis, and treatment. A literature review. Am J Orthod Dentofacial Orthop 2004;126(4):432-445.

25. Vaysse F, Noirrit E, Bailleul-Forestier I, Bah A, Bandon D. Les anomalies de l'éruption dentaire. Archives de pédiatrie 2010;17:756-757.

26. Wise GE. Cellular and molecular basis of tooth eruption. Orthod Craniofac Res 2009;12(2):67-73.

27. Wise GE, Frazier-Bowers S, D'Souza RN. Cellular, molecular, and genetic determinants of tooth eruption. Crit Rev Oral Biol Med 2002;13(4):323-334.

28. Yamaguchi T, et al. Exome resequencing combined with linkage analysis identifies novel PTH1R variants in primary failure of tooth eruption in Japanese. J Bone Miner Res 2011 ;26(7):1655-1661. 